ISBN 978-81-943403-5-5

18th ROME - ITALY International Conference on Art History, Literature, Social Sciences and

Education (AHLSE-20)

Rome (Italy) Feb. 3-5, 2020

\title{
The Role of Cross-linguistic Influence in Bilingual Children's Grammar Acquisition: A Review of Recent Research Evidence
}

\author{
Yiman $\mathrm{Yu}$ \\ The University of Warwick
}

\begin{abstract}
This paper aims to provide a critical account of some selected empirical works on cross-linguistic influences in bilingual children' s morpho-syntactic development. The review begins with a brief background introduction of several crucial issues in the field, then moves on to an in-depth discussion of several selected papers published within this decade, focusing on bilingual children speaking two typologically distant languages. According to this discussion, it can be concluded that findings on this topic are not completely homogeneous: majority of these studies have provided evidence for cross-linguistic transfers on bilingual children's grammar development along with several candidate explanations, including syntactic overlaps/ambiguities between bilinguals' two languages, processing competitions between the two syntactic systems in the bilinguals' brain, and language dominance effects. Nevertheless, no evidence for such influences was found in some studies, as exemplified by one in this review, suggesting that cross-linguistic influence may not be a phenomenon bound to general childhood bilingualism, but that its occurrence can be subject to a number of factors. Furthermore, both positive and negative cross-linguistic influence has been reported under different conditions, reflecting the ambivalent nature of this phenomenon, which should, therefore, be treated neutrally.
\end{abstract}

Keywords: critical review; cross-linguistic influencee; childhood bilingualism; syntax acquisition

\section{Introduction}

Considerable empirical evidence accumulated in early years has led to a relatively well-established consensus in bilingual language acquisition that bilingual children differentiate their two languages on a morpho-syntactic level from an early age (Meisel, 1994; De Houwer, 1990; Genesse, Nicoladis and Paradis, 1995; Koppe, 1996). Based on this consensus, the research focus has shifted to the issue of cross-linguistic influence in the last two decades. Specifically, researchers have been exploring under which circumstances, and to what extent the two grammatical systems held by bilingual children may come into contact and thus influence each other, generating quantitatively or qualitatively different linguistic behaviours compared to their monolingual counterparts. This paper aims to provide a sketch of the most recent development in this research area by selectively reviewing relevant empirical evidence published after the year 2010. I start by presenting working definitions of the key terms to demarcate the accurate sphere of this review and eliminate potential misconceptions, then move on to a brief recap of two prevailing theoretical accounts on this topic, which have laid solid groundwork for the recent works. The recent evidence are discussed in line with three major candidate explanations for cross- linguistic influence, which are grammatical overlaps and ambiguities, processing competitions, and language proficiencies respectively. By the end, I attempt to justify the inconsistencies displayed by all the studies in the discussion, as well as to identify potential gaps for future research.

\section{Definitions}

According to Jarvis (2012), cross-linguistic influence is often the preferred term for the phenomenon more commonly known as transfer. The former term is favoured in the literature arguably due to its wider cover range of cross-linguistic effects not only at the production level (including the over-usage, under-usage and avoidance 
of certain linguistic forms, structures or functions in one language driven by the influence from another); but also at the level of mental processing and conceptualization. Another frequently used sub-categorical term for cross-linguistic influence is interference, whose reference is limited to negative cross-linguistic influence as literally-implied. For the convenience of discussion, cross-linguistic influence as a generic phenomenon will be referred as CLI, CLI effects, or transfer interchangeably inattentive to any theoretical allegiance in the following discussion.

De Houwer (2009) defined childhood bilingualism as a linguistic phenomenon that transpired in children who have regular contact with two languages from early childhood up until the final stage of development. Yet there is a lack of consensus on the age threshold for either early-childhood or final stage of development in the literature possibly due to its high variability on an individual basis (Macleod, Fabiano-Smith, Beogner-Page \& Fontolliet, 2012). Since this paper is concerned with CLI observed in one's linguistically formative period instead of formulating a precise age threshold, a more flexible operating age range for bilingual children, known as the pre-puberty period (from birth up to age 12 years), is employed when selecting studies for the review.

\section{Hypotheses and Earlier Evidence}

Along the vein, considerable empirical evidence suggests that CLI is a systematic and predictable phenomenon that does not happen randomly, yet it appears more difficult to formulate general predictions, arguably because the cause, locus and direction of its occurrences are subject to several externally controlled factors, (e.g., language/linguistic domain specificity; language dominance; and age of acquisition). One early proposal holds that the direction of CLI is primarily influenced by the language dominance pattern, that is, bilinguals' dominant language usually functions as the 'model' language, whose syntactic properties are often used as templates for the acquisition of the counterpart structures in their weaker language, resulting in positive or negative transfer effects from the dominant language to weak one. Yet there has been counter-evidence suggesting that CLI does not always take place in this direction, and to explain these phenomena, a widely cited framework was proposed by Hulk and Müller (2000), as most accurately depicted in their own terms (Hulk \& Müller, 2000: 228-229):

Our hypothesis is that (syntactic) cross-linguistic influence occurs in bilingual children and that it is possible only if the two following conditions are both met:

1) Cross-linguistic influence occurs at the interface between two modules of grammar, and more particularly at the interface between pragmatics and syntax in the so-called $C$-domain, since this is an area which has been claimed to create problems in L1 acquisition also.

2) Syntactic cross-linguistic influence occurs only if Language A has a syntactic construction which may seem to allow more than one syntactic analysis and, at the same time, Language B contains evidence for one of these two possible analyses. In other words, there has to be a certain overlap of the two systems at the surface level.

Although logically complicated, these argumentations are clearly articulated and well instantiated with a series of case studies on several Germanic-Romance bilingual children acquiring grammatical structures that are either completely congruent (i.e., object drop), or only partially congruent (i.e., root infinitive) to the two proposed preconditions 11 (Müller \& Hulk, 1999; Müller, 1999; Hulk and Müller; 2000). Data analysis expectedly exhibit more omissions of obligatory objects in bilingual children's French and Italian speech

\footnotetext{
${ }^{1}$ Hulk and Müller (2000) explain that the phenomena of object drop and root infinitive both satisfy what is characterized in the first condition, since they are syntactically unanchored structures whose interpretations entail pragmatic information (i.e., discourse and other contextual cues). Yet contrary to the case of object drop (i.e., input of topic-drop Germanic languages reinforces the non-targetlike discourse licensing of dropping objects in Romance languages), root infinitive fail to meet the second condition because structural overlap between Romance and Germanic languages does not exist for this element, thus there is no possibility for one language to reinforce the misanalysis of structures in the other language.
} 
compared to that of their monolingual peers, whose cause is attributed to CLI effects from the bilingual children' knowledge of the Germanic language; albeit such quantitative difference (nor any qualitative difference indeed) is not found in terms of the production of root infinitives, based on which Hulk and Müller have drawn the central conclusion that the two conditions aforementioned are necessary yet may not be sufficient for CLI to occur. This conclusion has provoked a new research focus on identifying grammatical domains which are vulnerable to CLI.

Nevertheless, some subsequent studies with a similar experimental design fail to support this conclusion, since they find no significant difference between bilingual and monolingual children regarding their usages of syntactic properties that are supposedly vulnerable to CLI based on Hulk and Müller's hypothesis (e.g., JuanGarau \& Perez-Cidal, 2000; Catone \& Schmitz, 2001; Serratrice, 2002; Unsworth, 2003). Moreover, the scope of the hypothesis is also in dispute, as studies have demonstrated that CLI also affect children's acquisition of grammatical elements that lack morpho-syntactic overlap (Nicoladis, 2002; Yip \& Matthews, 2000), as well as the ones that do not tap into the syntax-pragmatics interface (Pannemann, 2006). In addition, it has been proven that the influence can occur even after the C-domain is set in place (Serratrice, Sorace \& Paoli, 2004). Although the scarcity of evidence does not necessarily invalidate the hypothesis per se, its practicality can still be undermined. Thus, more recent studies have been refining and substantiating these hypotheses with more rigorous methodologies and a wider array of language combinations and target grammatical structures to amply the breadth and depth of the investigation, as will be selectively reviewed in what follows.

\section{Recent Evidence}

Recent research where CLI is found in bilingual's grammar acquisition has identified several candidate driving forces, including the structural overlap and/or ambiguity between the two languages; the language dominance and/or proficiency; the processing competitions of the two grammatical systems. While other accounts have been proposed, these are the three that are most relevant for this paper and will be addressed in line with empirical evidence. To maintain a lucid structure, the three candidate predictors of CLI are used as subheadlines for the following sections to indicate the primary focus of each one; yet the headline does not prevent studies included in that section from briefly mentioning the other two predictors since the three predictors are not necessarily mutually exclusive, instead, they sometimes work conjointly and complementarily. Most studies to be discussed have identified more than one of them. Inter-sectional references might be drawn where relevant.

\subsection{Structural Ambiguities and Overlaps}

Inspired by the second condition in Hulk and Müller's hypothesis, structural ambiguities and overlaps between bilingual children's two languages have been confirmed as one major driving force for CLI effects in several recent studies. Chan's (2010) study further delves into this facet by investigating the acquisition of double- object dative construction in 7 Cantonese-English simultaneous bilinguals (aged 1;03-4;06 years) based in Hong Kong along with 8 Cantonese monolingual children as controls. The double-object dative is selected as the target structure since its acquisition has proven prone to errors and delays in both languages. Particularly, in addition to the V-G-T double-object structure captured in both languages, Cantonese also allows double-object datives with a reversed order of the two objects (V-T-bei2-G/V-T-G); theoretically, this potential overlap can lead to more structural variabilities and ambiguities in the bilingual input. Data was collected from participants' spontaneous speech in a naturalistic setting over three years. The result shows that bilingual children use nontarget- like Cantonese double-object datives (i.e., V-bei2-G-T) significantly more frequently and durably compared to the control group. Such difference is explained by analysis of the input received by bilinguals and monolinguals. Qualitatively, the inconsistencies between word order and thematic roles found in the Cantonese input makes the acquisition of the target structure intrinsically demanding; and for the bilingual children, the simultaneous exposure to the English input where double-forms are almost invariant has reinforced the V-G-T order, and it then negatively transferred to the target Cantonese structures. Quantitatively, the bilingual input contains considerably fewer tokens of the target structure (V-T-bei2-G) compared to that in the Cantonese 
monolingual input. It is thus concluded that the structural overlap and the reduced input conspire to make the acquisition of V-T-bei2-G a particularly vulnerable domain for CLI. One major contribution of this study is a thorough and systematic characterization of both the quantitative and qualitative aspects of the input bilingual children received in a specific grammatical domain, through which CLI and its potential driving force have been manifested. So far, it appears that Chan's conclusion neatly complies with Hulk and Müller's proposal, yet some key issues remain unaddressed due to the absence of English monolingual controls (e.g., the directionality of CLI).

Chan's finding concerning the role of structural ambiguity/overlap in causing CLI is further consolidated in Kidd, Chan and Chiu (2015)'s study from a different angle, where Cantonese-English bilingual children's comprehension of relative clauses (RCs) is under investigation. Though the canonical SVO word order exists in both Cantonese and English, English RCs are post-nominal as in contract to pre-nominal Cantonese RCs. Yet classifier-RC (CL-RC), a type of Cantonese object RCs often employed in an informal context, displays structural isomorphism to SVO clauses, creating structural ambiguities that are speculated to induce more comprehension difficulties for bilinguals. Methodologically, 20 Cantonese-English bilingual children (aged 4;10-11;11 years) along with 20 Cantonese-monolingual children within the same age and vocabulary-size range are recruited for RC comprehension testing (the bilinguals are required to take two tasks, a Cantonese one and an English one; whereas the monolinguals only take the Cantonese one). In general, the RC test scores were found positively correlated to the vocabulary scores. While the bilingual subjects significantly outperformed in comprehending subject RCs over object RCs, the monolinguals demonstrated balanced competences in both. Specifically, head- noun related errors in object RCs were most pervasive in both groups, yet the bilinguals' error rate here was three times that of the monolinguals'. Kidd et al. argued that this result could be explained by the Competition Model (c.f., Brain, 2002): the overlap between the transitive syntax of both languages (i.e., SVO) and the surface form of CL-RC has engendered competing parses in a bilingual brain, resulting in a more errored performance. Furthermore, regression analysis showed that the RC comprehension scores were predicted by the language dominance pattern at an individual level. Overall, evidence provided in this study is pregnant to the relevant research area: first, participants' vocabulary knowledge, in addition to a detailed demographic questionnaire about their linguistic background, were used to index their language proficiency (and thus language dominance pattern). While receptive vocabulary is an important measurement in this context, justifications of adopting this approach is necessary, since there turned out to be inconsistencies between the dominance pattern indicated by the vocabulary test scores (i.e., the bilinguals are equally proficient in English and Cantonese) and that indicated by the questionnaire results (i.e., the bilinguals are English-dominant), and this discrepancy was not resolved when they were establishing a predicting-relation between language dominance and CLI; second, the results have showed CLI can continue to affect the course of language development in older children aged beyond infancy and early childhood; third, the incorporation of the Competition Model in data interpretation is a plausible and intriguing attempt to enhance the predictability of the structural ambiguity/overlap account.

However, some studies have instantiated cases where CLI does not occur in cases where it could be predicted from syntactic ambiguities and overlaps. For example, Nicoladis, Rose and Foursha-Stevenson (2010)'s study observed French-English bilingual pre-schoolers' (aged 3;07-9;03) usage of constructions characterized by a co-existence of structural overlaps and conceptual discrepancies between the two languages. This conflicting condition was tactfully created with the choice of an action labeling task which induces two possible syntactic structures: nouns modified by relative clauses (NMRC) versus nouns modified by de-verbal adjectives (i.e., V- ing in English \& V-ant in French); despite the plausibility of both structures, French monolinguals demonstrate a strong preference of using NMRCs to foreground the moving objects, while deverbal adjectives are clearly favoured by English monolinguals to highlight the progressive action. Production data did not show evidence for CLI as the bilingual subjects behaved similarly with their monolingual counterparts in each respective language. Accordingly, overlaps on the conceptual level have been postulated as a pre-condition for structural ambiguities and overlaps to trigger CLI. Therefore, rather than invalidating Hulk 
and Müller's hypothesis, this study has introduced an extra layer of insight by tapping into the conceptual level of CLI, echoing the rationale of the Speech Production Model (c.f., Nicoladis, 2006).

\subsection{Processing Competitions}

Processing competitions, as another prevailing account for CLI, has been often discussed along with syntactic ambiguities/overlaps (e.g., Kidd et al., 2015; Nicoladis et al., 2010; Nicoladis, 2012), and Nicoladis (2011), some researchers therefore argued that processing competitions should not be viewed as an alternative to an explanation in terms of syntactic structures (e.g., Hulk \& Müller, 2000). Yet one recent evidence of CLI caused by processing competitions was reported in a deductive study of structural overlaps (Nicoladis \& Gavrila, 2015), primarily aiming to find out whether CLI would still take place when syntactic overlaps are absent. Accordingly, adjectival constructions in Welsh and English were selected as the target structures, since Welsh and English differ clearly regarding the placements of adjectives relative to nouns (i.e., adjectives are postnominal in Welsh and pre-nominal in English). Thirty Welsh dominant Welsh-English bilinguals (aged 3;00 to 6;00 years; language dominance pattern indexed by vocabulary tests) along with 28 English monolinguals undertook a picture-naming task designed to elicit production of the target structures. Contrary to what would have been predicted by Hulk and Müller's (2000) hypothesis, bilingual production data was marked with a significantly higher rate of reversed adjectival constructions in English compared to the monolingual one, when the reversal rates in Welsh and English are roughly identical, which was thus interpreted as evidence for CLI. Since structural overlaps have been purposefully eliminated by the experimental design, researchers proposed an alternative explanation for the observed CLI: the processing constraints aroused from competitions between two language systems. In other words, bilingual children have access to more than one grammatical structures mapped onto a same meaning, among which the interferences between optimal and sub-optimal options at the syntactic level result in speech errors manifested as reversals of adjectives and nouns for the target language (i.e., CLI). The explanation has been further supported by the fact that most adjectival constructions produced by the bilingual subjects in both languages were identical with the canonical word order of the target language. Despite the contribution to the literature where CLI occurs without overlaps, this paper did not explicitly formulate any generalizable conditions under which CLI would be triggered by processing competitions based on its finding (although language dominance and age were suspected as predictors).

\subsection{Language Proficiency (Dominance Pattern and Input Quantity)}

In BFLA, children's language proficiency is usually measured by either receptive vocabulary (often used as the predictor for language dominance), or language input quantity. Despite being positively correlated, these two measurements have been designed to address different aspects of language proficiency (i.e., the vocabulary measurement taps specifically into lexical skills while the input quantity predicts more general linguistic abilities), and both approaches have been frequently tested as potential predictors for CLI. Apart from what has been discussed in the last section, Nicoladis and Gavrila (2015)'s study also aims to explore the relationship between language dominance and CLI. It was hypothesized that this relationship would be translated to a negative correlation between the vocabulary scores and the rate of reversals of the bilingual subjects. ANOVA analysis showed that while the negative correlation between Welsh vocabulary scores and reversal rates in Welsh did not achieve significance, no correlation was found between English vocabulary scores and reversal rates in either language, adding evidence to the growing belief that CLI cannot be predicted by the language proficiency measured in terms of receptive vocabulary alone (Gathercole \& Holf, 2007; Hsin, Legendre \& Omak; 2013; Nicoladis, 2012). Yet Nicoladis and Gavrila were more cautious when making claims about individual cases, since the bilingual children were Welsh-dominant as a group, and they produced a statisticallyinsignificant higher proportion of English reversals compared to their monolinguals counterparts, suggesting a potential role language dominance pattern may play in causing CLI, but only at a group level. The gap regarding language dominance effects on an individual basis has been filled by the findings in Kidd, Chan and Chiu's study (2015) as discussed above (c.f., page 4-5). Despite the different findings, both studies quest important 
follow-ups to test a larger group of children and with a wider array of linguistic profiles, as well as monolingual controls in both studied languages, before drawing a more generalizable conclusion.

In term of input quantity, Herve, Serratrice and Corley (2016) explored its potential impact on the degree of accessibilities of the cross-linguistic syntactic structures which in turn would predict CLI. Specifically, 38 French-English bilingual children aged between 5;04 and 6;07 years were recruited as research subjects, their frequency (i.e., marked in ratio) of applying the left dislocation (LD) structures in the picture description tasks was compared to that of 20 monolingual counterparts in each language. This particular target structure has been chosen because it captures not only cross-linguistic pragmatic-syntactic overlaps (i.e., LDs mark topics in both languages), but also different accessibilities (i.e., French LDs are discourse-old or inferable referents whose usage is prevailing; whereas English LDs introduce discourse-new ones whose usage is relatively much rarer) between the two target languages. Moreover, the input quantity bilingual children received in each language was estimated on an individual basis according to the information provided by their parents in an exhaustive questionnaire, asking for details of their daily language use. Results showed that the relative quantity of input bilingual subjects received in each language did predicate their likelihoods to produce LDs in both languages, adding another piece of supporting evidence for the recent finding that the magnitude of CLI alters as a function of the bilingual children's exposure to their language input (Serratrice, Sorace, Filiaci, \& Baldo: 2009; 2011). This study is characterized by an exceptionally rigorous methodological deign, especially regarding the prudent quantifiable-measurement of the input quantity based on individual questionnaire data instead of making hasty assumptions according to the language of the community. This adaption has proven necessary as the quantified questionnaire data demonstrated that the predominant input exposure a subject got was not necessarily in the language of the local environment. Therefore, findings in previous studies where the local community language was used as a proxy for the input quantity risk bearing confounding effects from a falsely assumed language exposure pattern.

\section{Concluding Remarks}

This paper has selectively discussed several key research evidence on CLI accumulated in the last few years in line with three highly endorsed candidate explanations for such influence: namely structural ambiguity and overlap; processing competition and language proficiency effects. Compared to the earlier evidence, the studies under discussion have tapped into more depth and breadth on this topic, reflecting the maturational process of research in this area. Specifically, the investigation has been extended to a wider array of language pairs which greater typological distance (e.g., Cantonese-English); the methodologies have been more tactfully manipulated to control more confounding variables; furthermore, the nature of the recent studies is becoming increasingly interdisciplinary, for instance, several studies have attempted to deepen the insight on CLI by adapting relevant psycholinguistic theories to explain the observed linguistic phenomenon on a cognitive level.

However, as evidenced in the discussion, even studies with similar focuses do not display completely consistent findings, corroborating to the intrinsically complex and specific nature of CLI. Most studies selected for this review have demonstrated evidence for CLI, yet its causes, directions and ways of manifesting could differ from case to case. So far, it is not feasible to draw a universally applicable account for all disparities observed across the studies, particularly considering the fact that we have not yet come up with a delimited condition for CLI to always take place. Since this paper is only a selective review of the recent evidence, the real picture in this field is even more complicated than what has been displayed above. For example, apart from the three predictors discussed in the paper, CLI has also been proven vulnerable to a number of other factors, such as the particular grammatical features in a language and the age of acquisition, whose interpretation entails a closer examination of each concrete case. Moreover, there has also been a reasonable amount of studies in the literature where CLI was not attested. Instead, alternative explanations from other linguistic perspectives were proposed for the differences observed between bilinguals and monolinguals' grammar acquisition, such the immature language strategy adopted by the bilinguals, bilinguals' naturally higher pragmatic sensitivity, the nature of 
bilingualism, or the lack of a conceptual overlap for the target structure between two languages, all suggesting that CLI is not a bound phenomenon to childhood bilingualism (e.g., Barreba \& Almgren, 2013; Mykhayyk, 2013; Pirvulescu, Pérez -Leroux, Roberge, Strik \& Thomas 2013). And when extending the bilingual population across a wide age span, the most prevailing account for CLI so far, the syntax-pragmatic interface vulnerability one, have been empirically challenged on different levels. For instance, Cuza (2012)'s study provided clear evidence of CLI on the acquisition of a core syntax structure without any pragmatic constraints (i.e., interrogative subject-verb inversion) even in Spanish-English adult bilinguals. Considering theoretical aspect of this issue, it seems that the puzzle of CLI still needs many more pieces from different angles to be completed. On the other hand, as exemplified in the discussion, CLI on bilingual children's grammar acquisition can be either facilitating or impeding depending on specific cases, future studies may give more prominence to pedagogical implications where relevant to offer scientific guidelines for bilingual children's language acquisition.

\section{References}

[1] Barrena, A. and Almgren, M. "Object-verb and verb-object in Basque and Spanish monolinguals and bilinguals," International Journal of Bilingualism, 17(3), pp. 337-356, 2012.

https://doi.org/10.1177/1367006912438993

[2] Cantone, K. F. \& Schmitz, K, "Subject and object omission in a German-Italian bilingual child," In Colloquium on Structure, Acquisition and Change of Grammars: Phonological and Syntactic Aspects, K. F. Cantone \& M.-O. Hinzelin, Ed. . Hamburg: Sonderforschungsbereich 538 Mehrsprachigkeit, 2001, ch.1, pp.20-36.

[3] Chan, A., "The Cantonese double object construction with bei2 'give' in bilingual children: The role of input," International Journal of Bilingualism, 14(1), pp. 65-85, 2010.

https://doi.org/10.1177/1367006909356653

[4] Cuza, A., "Cross-linguistic influence at the syntax proper: Interrogative subject-verb inversion in heritage Spanish," International Journal of Bilingualism, 17(1), pp. 71-96, 2013.

https://doi.org/10.1177/1367006911432619

[5] De Houwer, A., The acquisition of two languages from birth: A case study, Cambridge, Cambridge: Cambridge University Press, 1990.

https://doi.org/10.1017/CBO9780511519789

[6] ----, Bilingual first language acquisition, Bristol, UK: Multilingual Matters, 2009.

[7] Hulk, A. and Müller, N., "Bilingual first language acquisition at the interface between syntax and pragmatics," Bilingualism, 3(3), pp. 227-244, 2000.

https://doi.org/10.1017/S1366728900000353

[8] Gathercole, V.C.M., \& Hoff, E., "Input and the acquisition of language: Three questions," in Blackwell handbook of language development, E. Hoff \&M. Shatz, Ed. Oxford: Blackwell Publishing, 2007, pp. 107-127.

https://doi.org/10.1002/9780470757833.ch6

[9] Genesee, F., Nicoladis, E., \& Paradis, J., "Language differentiation in early bilingual development," Journal of Child Language, 22, pp. 611-631, 1995.

https://doi.org/10.1017/S0305000900009971

[10] Herve, Serratrice \& Corley, "Dislocation in French and English bilingual children: An elicitation study," Bilingualism: Language and Cognition, 19 (5): 987-1000, 2016.

https://doi.org/10.1017/S1366728915000401

[11] Hsin, L., Legendre, G., \& Omaki, A., "Priming cross-linguistic interference in Spanish-English bilingual children," Presented at the proceedings of the 37th Annual Boston University Conference on Language Development. 
[12] Jarvis, S., "Cross-linguistic Influence and Multilingualism," in The Encyclopaedia of Applied Linguistics, C.A. Chapelle Ed. Boston: Wiley Blackwell Press, 2012.

https://doi.org/10.1002/9781405198431.wbeal0291

[13] Juan-Garau, M. \& Pe ŕ ez-Vidal, C., "Subject realisation in the syntactic development of a bilingual child," Bilingualism: Language and Cognition, 3, PP. 173-192, 2000.

https://doi.org/10.1017/S1366728900000328

[14] Kidd, E., Chan, A. \& Chiu, J., "Cross-linguistic influence in simultaneous Cantonese \& English bilingual children's comprehension of relative clauses," Bilingualism, 18(03), pp. 438-452, 2015.

https://doi.org/10.1017/S1366728914000649

[15] Koppe, R., "Language differentiation in bilingual children: the development of grammatical and pragmatic competence," Linguistics, 34, pp. 927-954, 1996.

https://doi.org/10.1515/ling.1996.34.5.927

[16] Meisel, J., “Code-Switching in Young Bilingual Children” Studies in Second Language Acquisition, 16(04), pp. 413, 1994.

https://doi.org/10.1017/S0272263100013449

[17] Müller, G., "Imperfect checking," The Linguistic Review, 16(4), 1999.

https://doi.org/10.1515/tlir.1999.16.4.359

[18] Mykhaylyk, R., "Bilinguals vs. monolinguals: Where is the difference?," Studia Linguistica, 67(1), pp.101- 122, 2013. https://doi.org/10.1111/stul.12007

[19] Nicoladis, E., "What's the difference between 'toilet paper' and 'paper toilet'? French-English bilingual children's crosslinguistic transfer in compound nouns," Journal of Child Language, 29(4), 2002. https://doi.org/10.1017/S0305000902005366

[20] ----, “Cross-linguistic transfer in adjective-noun strings by preschool bilingual children," Bilingualism: Language and Cognition, 9 (1), pp. 15-32, 2012.

https://doi.org/10.1017/S136672890500235X

[21] ----, "Cross-linguistic influence in French \& English bilingual children's possessive constructions," Bilingualism, 15(02), pp. 320-328, 2012.

https://doi.org/10.1017/S1366728911000101

[22] Nicoladis, E. and Gavrila, A., "Cross-linguistic influence in Welsh \& English bilingual children's adjectival constructions," Journal of Child Language, 42(04), pp. 903-916, 2015.

https://doi.org/10.1017/S0305000914000440

[23] Nicoladis, E., Rose, A. and Foursha-Stevenson, C., "Thinking for speaking and cross-linguistic transfer in preschool bilingual children," International Journal of Bilingual Education and Bilingualism, 13(3), pp. 345-370, 2010. https://doi.org/10.1080/13670050903243043

[24] Pannemann, M., "More variability in French L1: consequences for theories of DP-acquisition," in Linguistik International 16. Variation in Sprachtheorie und Spracherwerb, M. Vliegen Ed. Amsterdam: Lang, 2006, pp. 223-232.

[25] Pirvulescu, M., Pérez -Leroux, A., Roberge, Y., Strik, N. and Thomas, D., "Bilingual effects: Exploring object omission in pronominal languages," Bilingualism, 17(03), pp. 495-510, 2013.

https://doi.org/10.1017/S1366728913000631

[26] Serratrice, L., "Overt subjects in English: Evidence for the marking of person in one English-Italian bilingual child," Journal of Child Language, 29, pp. 1-29, 2002.

https://doi.org/10.1017/S0305000902005068 
[27] Serratrice, L., Sorace, A. \& Paoli, S., "Cross-linguistic influence at the syntax-pragmatics interface: Subjects and Objects in English-Italian bilingual and monolingual acquisition," Bilingualism: Language and Cognition, 7(3), pp. 183-205, 2004.

https://doi.org/10.1017/S1366728904001610

[28] Serratrice, L., Sorace, A., Filiaci, F., \& Baldo, M., "Bilingual children's sensitivity to specificity and genericity: Evidence from metalinguistic awareness," Bilingualism: Language and Cognition, 12(2), pp. 239-257, 2009. https://doi.org/10.1017/S1366728909004027

[29] ----, "Pronominal objects in English-Italian and Spanish- Italian bilingual children," Applied Psycholinguistics, 33(4), pp. 725, 2011. https://doi.org/10.1017/S0142716411000543

[30] Unsworth, S., "Testing Hulk \& Müller (2000) on cross-linguistic influence: RIs in a bilingual German/English child," Bilingualism: Language and Cognition, 6(2), pp. 143-158, 2003.

https://doi.org/10.1017/S1366728903001093

[31] Yip, V. and Matthews, S., "Syntactic transfer in a Cantonese \& English bilingual child," Bilingualism, 3(3), pp. 193208, 2000.

https://doi.org/10.1017/S136672890000033X 